\title{
Quantum Theory of the Wannier Phenomenon
}

\author{
Hubert Klar \\ Retired from University of Freiburg, Freiburg im Breisgau, Germany \\ Email: hubklar@aol.com
}

How to cite this paper: Klar, H. (2020) Quantum Theory of the Wannier Phenomenon. Journal of Applied Mathematics and Physics, 8, 2416-2426. https://doi.org/10.4236/jamp.2020.811178

Received: September 13, 2020

Accepted: November 13, 2020

Published: November 16, 2020

Copyright (c) 2020 by author(s) and Scientific Research Publishing Inc. This work is licensed under the Creative Commons Attribution International License (CC BY 4.0).

http://creativecommons.org/licenses/by/4.0/

\begin{abstract}
We employ a recently amended Born-Oppenheimer (hereafter shortly $\mathrm{BO}$ ) approximation [1] to treat inelastic scattering of slow electrons from highly excited Rydberg atoms like $\mathrm{e}^{-}+\mathrm{He}(1 s n s) \rightarrow \mathrm{He}^{-* *}$ for $n \gg 1$. Along these lines we replace the standard BO set of potentials by an evolution operator. In this way we take a momentum-momentum coupling inadvertently disregarded by BO into account. The BO eigenvalue problem is now replaced by an evolution equation. One eigen-evolution has been identified as Wanner channel. That channel describes the diffraction of electron pairs from a potential ridge. That diffraction causes a phase jump of $\pi / 2$ in the channel evolution. Moreover we present a new conservative attractive force controlling the motion of the electron pair as a whole in the nuclear field whose potential is given by $W(R)=-\frac{g}{R^{3 / 2}}$. The coupling constant $g$ has been calculated. That potential foreign to the standard $\mathrm{BO}$ approximation manifests itself by an entirely new series of isolated resonances located slightly below the double ionization threshold. This resonance ensemble compares favorably with experimental data. Further we present an evolution which forces the electron pair to the electrostatically unstable top of the potential ridge. That evolution may be regarded as quantum version of Wannier's converging trajectory, and manifests itself here as Fresnel distribution.
\end{abstract}

\section{Keywords}

Born-Oppenheimer, Wannier Theory, Non-Separable Wave Equations

\section{Introduction}

In 1953 Wannier surprised the atomic community with an entirely unusual threshold ionization law for electron impact ionization of the hydrogen atom [2]. On the basis of classical mechanics he came to a power law with fractional exponent, given by 


$$
\sigma \propto E^{1.127 \cdots} .
$$

In the meantime there exists highly accurate experimental data which confirm that exponent (see [3] and references therein).

Wannier's picture is basically as follows. As long as the scattering electron is still away from the target atom the bound electron performs periodic Bohr orbits. As soon as the incident electron comes closer to the target these Bohr orbits experience a strong distribution due to correlation. At very low energy the electron-electron repulsion forces the three bodies into a collinear configuration (electron-nucleus-electron). Within that collinear configuration the electron pair experiences an unstable equilibrium position when both electrons have equal distances from the nucleus, say $r_{1}=r_{2}$. Actually a cut through the potential surface,

$$
V=-Z\left(\frac{1}{r_{1}}+\frac{1}{r_{2}}\right)+\frac{1}{r_{12}}
$$

shows a repulsive barrier whose top is located at $\frac{r_{1}}{r_{2}}=1$. In (2) $r_{12}$ is the electron-electron separation. Wannier has therefore introduced an angular coordinate

$$
\alpha=\tan ^{-1} \frac{r_{1}}{r_{2}}
$$

and studied the Langrage equations in the subspace $(R, \alpha)$ where

$$
R=\sqrt{r_{1}^{2}+r_{2}^{2}}
$$

is the so called hyperradius orthogonal to $\alpha$. That subspace constitutes geometrically a ridge. Unfortunately, the coordinates $R, \alpha$ are neither in the classical Hamilton function nor in its quantum counterpart separable. Nevertheless, Wannier was able to show the existence of two special trajectories during the evolution along $R$. One trajectory converges to the top of the ridge whereas the other one diverges away from it. It is the aim of the present paper to present a mathematical frame within quantum theory which delivers two kinds of wave propagation which reduce in the classical limit to Wannier's trajectories.

We do that in two steps. First we introduce an angle different from Wanniers $\alpha$ (see Section 2). Our angle (below denoted by $\varphi$ ) may be obtained by a rotation in the space $\mathbb{R}_{6}$ such that the better suited angle $\varphi$ becomes a cyclic coordinate (see for instance [4]). Inspection of the Hamiltonian in hyperspherical coordinates shows that the whole system depends only smoothly on the coordinate $R$. That observation invites us to apply a $\mathrm{BO}$ approximation where $R$ is the adiabatic coordinate. That procedure was used already many years ago by Macek [5], later also by us [6]. But the BO approximation does not deliver the wave propagations we are looking for. The reason is a fundamental mistake in the $\mathrm{BO}$ approximation. The author showed recently [1] that $\mathrm{BO}$ has inadvertently disregarded a portion of the kinetic energy, namely a momentum-momentum coupling. Ref [1] avoids that shortcoming, and derives a new evolution equation 
replacing the $\mathrm{BO}$ eigenvalue problem by an evolution equation. It is the aim of this paper to transfer our molecular method to electron-atom/ion scattering in the critical spectral range near ionization. Along these lines we discover the desired Wannier mode of motion as one particular eigen-evolution. Section 3 develops the amended $\mathrm{BO}$ technique for the present application, and finally we treat the discussion of the resonance spectrum of two-electron atoms close to the threshold of double escape (see Section 4). Section 5 summarizes our conclusions.

\section{The Hyperspherical Description of Atoms}

Wannier [2] has demonstrated why hyperspherical coordinates are particularly useful to describe collective motions of atomic electrons. The reason is as follows: Let us consider an atom with $N$ electrons. It is trivial to describe their positions in a hypersphere, i.e. we introduce a hyperradius now for $N$ electrons given by

$$
R=\sqrt{\sum_{i=1}^{N} r_{i}^{2}}
$$

and complete that generalized coordinate with $3 N-1$ hyperpolar angles corresponding to unit vectors on the hypersphere $\mathcal{S}_{3 N-1}$. Each single electron position vector has then the form

$$
\boldsymbol{r}_{i}=R \hat{R}_{i}(\omega)
$$

where $\omega$ stands for the set of angles, and $\hat{R}$ is a unit vector depending on these angles. It is then straightforward to see that the Hamiltonian of any atom has in non-relativistic description the structure

$$
H=T_{R}+\frac{\Lambda^{2}}{2 R^{2}}+\frac{C(\omega)}{R} .
$$

where $\Lambda^{2}$ is the Casimir operator of the rotation group $\mathrm{SO}_{6}$ and $C(\omega)$ is the potential multiplied by $R$.

In the case of two electrons Wannier pointed out that (7) at zero-energy obeys a similarity principle which describes classical trajectories in which the whole electron cloud performs at a set of constant angles hyperradial breathing motions. This requires of course that the electron cloud has an equilibrium configuration. Actually this is the case for few-electron atoms (see [4]). These motions including their stability constitute the so-called zero-energy Wannier phenomenon we are looking for.

The case of two electrons in the field of one nucleus has been worked out in great detail. We follow here Klar et al. [6]. For the description of the electrons we need five angles. Three of them may be chosen as Euler angles to describe the overall rotation of the atom in space. Since that rotation has nothing to do with the Wannier phenomenon we are looking here only for $S$-states. That reduces the number of necessary body-fixed angles to two.

One of them we chose to describe the relative motion within the collinear configuration (electron-nucleus-electron). Following Sommerfeld [7] we intro- 
duce in the collinear configuration the hyperspherical azimuth angle already mentioned above

$$
\varphi=\tan ^{-1} \frac{r_{1}^{2}-r_{2}^{2}}{2 r_{1} r_{2}}
$$

$(0 \leq \varphi \leq 2 \pi)$ whereas the bending motion may be described by the ratio of moments of inertia,

$$
\psi=\frac{1}{2} \tan ^{-1}\left(\sqrt{\frac{\Theta_{x x}}{\Theta_{y y}}}\right) .
$$

Macek [5] used instead of our $\varphi$ the Wannier angle $\alpha=\tan ^{-1} \frac{r_{1}}{r_{2}}$. Our angle $\varphi$, however, has the advantage to be a cyclic coordinate in the kinetic energy expression, whereas $\alpha$ is not.

The equilibrium configuration occurs in terms of our coordinates at $\psi=0$ corresponding to the collinear configuration and at $\varphi=\pi$ corresponding to equal electron-nucleus separations $r_{1}=r_{2}$. Since the Wannier phenomenon occurs only in the subspace $(R, \varphi)$ we fix below the angle $\psi=0$.

\section{Amended Born-Oppenheimer Approximation Applied to Excitation and Ionization of $\boldsymbol{H}$ by Electrons}

Although the standard BO approximation [8] was developed for molecules, that method appears suitable to treat also other non-separable systems like electron-atom/ion collisions [5] [6]. The pioneer work by Macek [5] has demonstrated that a small parameter like a mass ratio (electron to nucleus mass) is not necessary for the BO validity. Actually it is the smoothness of the Coulomb potentials which justifies the $\mathrm{BO}$ approximation, also referred to as adiabatic approximation. The $\mathrm{BO}$ technique applied to $\mathrm{He}$ and $\mathrm{H}^{-}$delivers indeed good results for singly and doubly excited states. The method fails, however, to describe the Wannier phenomenon. This is not surprising if the hyperradius $R$ is selected as adiabatic coordinate. In the $\mathrm{BO}$ approximation the hyperradius $R$ is treated as a constant in the channel expansion.

Actually, the standard $\mathrm{BO}$ approximation suffers from a serious mistake. It is usually believed that the validity rests on a small radial momentum in our present problem given by

$$
p=\frac{1}{i} \frac{\mathrm{d}}{\mathrm{d} R} .
$$

That is not true, neither in the molecular nor in the atomic case. The small quantity for the definition of a collision channel is the radial kinetic energy given by

$$
T_{R} \propto \frac{1}{2} P^{2}=-\frac{1}{2} \frac{\mathrm{d}^{2}}{\mathrm{~d} R^{2}}
$$

We look now to the Hamiltonian applied to a product wave function, see (6), 


$$
\begin{aligned}
H\{F(R) \Phi(\omega ; R)\} & =\frac{1}{2}\left(P^{2} F\right) \Phi+(P F)(P \Phi)+\frac{1}{2} F\left(P^{2} \Phi\right)+F h_{a n g} \Phi \\
& =-\frac{1}{2} \frac{\mathrm{d}^{2} F}{\mathrm{~d} R^{2}} \Phi-\frac{\mathrm{d} F}{\mathrm{~d} R} \frac{\partial \Phi}{\partial R}-\frac{1}{2} F \frac{\partial^{2} \Phi}{\partial R^{2}}+F h_{\text {ang }} \Phi
\end{aligned}
$$

where the angular Hamiltonian reads, see [6]

$$
h_{\text {ang }}=\frac{\Lambda^{2}}{2 R^{2}}+\frac{C(\omega)}{R} \text {. }
$$

BO introduces now mistakenly an adiabatic Hamiltonian disregarding the term $P \Phi$. That corresponds to the assumption of constant values of $R$. It is obvious that this primitive approximation cannot deliver the Wannier mode since already in Wannier's classical treatment $R$ is a function of time. We need therefore an improvement which allows for variable values of $R$.

Recently [1] we went one step ahead introducing radial zero-energy channels by asking $\mathrm{d}^{2} F / \mathrm{d} R^{2}$ to be the small quantity. That non-adiabatic description rejects only the channel coupling term $\partial^{2} \Phi / \partial R^{2}$ identified by Macek as coupling operator $Q$. To this end we arrive at the amended channel operator

$$
\left\{(P F) P+F h_{\text {ang }}\right\} \Phi \text {. }
$$

For a finite radial function $F \neq 0$ that leads to post-adiabatic eigen-channels defined by

$$
\left\{-\frac{\mathrm{d} F}{\mathrm{~d} R} \frac{\partial}{\partial R}+h_{\text {ang }}\right\} \Phi=W \Phi
$$

Before we go more deeply into the new development we review briefly the application of the standard BO approximation to two-electron atoms. After splitting off a factor of $R^{\frac{5}{2}}$ from the wave function the time-independent wave equation shows in hyperspherical coordinates the structure

$$
\left(-\frac{\partial^{2}}{\partial R^{2}}+\frac{15}{8 R^{2}}+\frac{\Lambda^{2}}{2 R^{2}}+\frac{C(\psi, \varphi)}{R}-E\right) \Psi(R, \psi, \varphi)=0
$$

see [6] for details. Following [8] and Macek [5] we try to construct the solution in product form

$$
\Psi(R, \omega)=F(R) \Phi(\omega ; R)
$$

where the channel function $\Phi$ solves (15) at constant values of $R$, i.e.

$$
\left(\frac{15}{8 R^{2}}+\frac{\Lambda^{2}}{2 R^{2}}+\frac{C(\psi, \varphi)}{R}\right) \Phi=U \Phi .
$$

The separation parameter $U$ constitutes in the case of helium for example an e- $\mathrm{He}^{+}(\mathrm{nl})$ potential. The channel function $\Phi$ represents standing waves on the hypersurface $\mathbb{S}_{5}$. The Wannier mode, however, we expect to be a travelling wave, and therefore is not member of the adiabatic channel family.

The present sketch derivation above suffers from an incorrect separation of variables. 
The zero-momentum approach identical to the standard BO approximation delivers for two-electron atoms good results for the lowest channels. But it becomes shaky for increasing excitation, and breaks down near threshold of double escape. Directly at threshold the classical Wannier modes of motion show a finite radial velocity $\dot{R}(t) \neq 0$, see [2]. We need therefore an improved description beyond a zero-momentum approach. To this end we employ the amended channel Equation (15). There we are, however, confronted with a further difficulty. The separation of variables is still incomplete because of the presence of the radial function $F(R)$ which is still unknown. Fortunately we need only its derivative $\mathrm{d} F / \mathrm{d} R$. That invites us to apply a trick introduced long ago by Sommerfeld $^{1}$, and expresses the derivative by its logarithmic one, i.e.

$$
F^{\prime}=\frac{F^{\prime}}{F} F=\frac{\mathrm{d}(\log F)}{\mathrm{d} R} F=i P(\log F) F
$$

The logarithmic derivative, moreover, we use to define collision channels, in our case

$$
P(\log F)=K(R)
$$

$K$ being the hyperradial wavenumber. That trick leads us to the channel equation

$$
\{(P F) P+F h\} \Phi=F\left\{i K \frac{\partial}{\partial R}+h(\omega ; R)\right\} \Phi=F W(R) \Phi
$$

which reduces for a finite radial function $F \neq 0$ to

$$
\left\{i K(R) \frac{\partial}{\partial R}+h(\omega ; R)\right\} \Phi=W(R) \Phi
$$

The radial wavenumber is given by twice the radial kinetic energy, expressed by total energy minus potential energy,

$$
K(R)= \pm \sqrt{2(E-W(R))}
$$

Equation (22) constitutes therefore a nonlinear eigenvalue equation because the lhs of (22) depends on the eigenvalue $W$, too. The quantity in curly brackets of (22) resembles a time-dependent wave equation where time has been replaced by radius. Like the time-dependent wave equation or the heat equation or our (22) describes an evolution. Therefore (22) may be rewritten as evolution Equation. To this end we introduce an evolution operator $E(\omega \mid R, 0)$ which describes the evolution from the united atom corresponding to $R=0$ to a finite value $R \neq 0$. Let us denote the channel function at $R=0$ with $\Phi(\omega ; 0)$ we put

$$
\Phi(\omega ; R)=E(\omega \mid R, 0) \Phi(\omega ; 0)
$$

with the initial condition

$$
E(\omega \mid 0,0)=1
$$

${ }^{1}$ Sommerfeld [7] uses that in the context of emitted radiation. We do it here for electrons. 
The evolution operator itself satisfies then an equation identical to (22)

$$
\left(i K \frac{\mathrm{d}}{\mathrm{d} R}+\frac{15}{8 R^{2}}+\frac{\Lambda^{2}}{2 R^{2}}+\frac{C(\psi, \varphi)}{R}\right) E(\omega \mid R, 0)=W(R) E(\omega \mid R, 0) .
$$

With help of the wavenumber $K$ we are now able to introduce collision channels, for instance by the energy level of the hydrogen-like target, here $\mathrm{He}^{+}$, with energy levels

$$
E_{n}=-\frac{Z^{2}}{2 n^{2}}
$$

In the following, we intent to treat also nuclei with arbitrary charges $Z$.

The wavenumber $K$ in (23) reads in the present case, see [1]

$$
K=K_{n}= \pm \sqrt{-\frac{Z^{2}}{2 n^{2}}-W(R)}
$$

The wavenumber in the Wannier spectral range needs a modification. The zero-energy domain is there controlled by a long-range Coulomb force. Note, at threshold $E=0$ the Coulomb zone extends to $R=\infty$. The Equation (28) needs therefore a modification since we expect $W(R)$ to be short-range potential. We proceed as follows.

Near threshold we expect the electron pair to be located near the ridge top. Therefore we approximate the ridge top by a Taylor expansion,

$$
C(\psi=0, \varphi)=-C_{0}-C_{2}(\varphi-\pi)^{2}+O(3) .
$$

The coefficients are given by

$$
C_{0}=\frac{4 Z-1}{\sqrt{2}}
$$

equal to the net charge of the correlated electron pair on the ridge top, and the ridge curvature

$$
C_{2}=\frac{12 Z-1}{4 \sqrt{2}}
$$

(29) identifies a long-range interaction representing a Coulomb potential in the space $\mathbb{R}_{6}$. i.e. the correlated electron pair as a whole with a charge $C_{0}$ is attracted by the nucleus. Thus we have to replace the eigenvalue $W$ in (28) by $-C_{0} / R$. Along these lines and using (28) the Wannier wavenumber becomes

$$
\lim _{n \rightarrow \infty} K_{n}=K_{\infty}=\sqrt{\frac{2 C_{0}}{R}}
$$

Finally, we need the operator $\Lambda^{2}$. That reads in the frame of our model at fixed $\psi=0$ simply

$$
\Lambda^{2}=-4 \frac{\partial^{2}}{\partial \varphi^{2}}
$$

since the azimuth angle $\varphi$ is a cyclic coordinate, see [4] [6]. The Wannier channel evolution equation to be treated reads therefore 
$\left\{i \sqrt{\frac{2 C_{0}}{R}} \frac{\partial}{\partial R}+\frac{15}{8 R^{2}}-\frac{2}{R^{2}} \frac{\partial^{2}}{\partial \varphi^{2}}-\frac{C_{2}}{R}(\varphi-\pi)^{2}\right\} E(\varphi \mid R, 0)=W(R) E(\varphi \mid R, 0)$

The following Section 4 solves that equation (34) and discusses the result.

\section{The Wannier Evolution}

We solve now the partial differential Equation (34) in the Coulomb zone employing the initial condition $E(\varphi \mid 0,0)=1$. To this end we disregard the centrifugal term, and try the exponential Ansatz

$$
E=\exp \left\{i \gamma g(R)(\varphi-\pi)^{2}\right\}
$$

with $g(0)=0$ and a constant $\gamma \in \mathbb{C}$. Along these lines we conclude immediately $g(R)=\sqrt{R}$ provided the constant $\gamma$ satisfies the quadratic equation

$$
8 \gamma^{2}-\sqrt{\frac{C_{0}}{2}} \gamma-C_{2}=0
$$

Equation (36) may be interpreted as description of a potential surface curvature deformation.

The solution (35) delivers for the rhs of (34) $\frac{4 i \gamma}{R^{3 / 2}} E$. We stress that the Hermiticity of the lhs of (34) excludes an imaginary eigenvalue. We conclude therefore that the diffraction of the correlated two-electron wave from the ridge has caused a phase jump ${ }^{2}$ of $\frac{\pi}{2}$, and forces the rhs of (34) to the interpretation

$$
W \exp \left\{i\left(\frac{\pi}{2}+\gamma \sqrt{R}(\varphi-\pi)^{2}\right)\right\}
$$

with the real eigenvalue

$$
W(R)=\frac{4 \gamma}{R^{3 / 2}}
$$

provided $\gamma$ is real. Actually we get the from (35) two real solutions given by

$$
\gamma_{1,2}=\frac{\sqrt[4]{2}}{32}(\sqrt{4 Z-1} \pm \sqrt{100 Z-9})
$$

where we have used (30), (31).

The existence of two solutions is in agreement with Wannier's observation. He got one trajectory converging to the equilibrium point $\varphi=\pi$ and one diverging away from it. Our positive $\gamma$ value describes indeed an evolution converging to the ridge top as may be seen as follows. The evolution (35) constitutes a Fresnel distribution with the property

$$
\lim _{R \rightarrow \infty} \exp \left\{i \gamma \sqrt{R}(\varphi-\pi)^{2}\right\}=\sqrt{\frac{i \pi}{\gamma}} R^{-\frac{1}{4}} \delta(\varphi-\pi) E(\varphi=\pi ; R \gg 1) .
$$

Here we have used the definition of a Fresnel Distribution given by

${ }^{2}$ Such a phase jump was also observed by Fano [10] in his resonance theory. 


$$
\Delta_{\text {Fresnel }}=\sqrt{\frac{a}{i \pi \eta}} \exp \left\{\frac{i a x^{2}}{\eta}\right\}
$$

valid for $x$ real and real $a>0$, see [9].

The diverging solution $(\gamma<0)$ leads Wannier's study to double electron escape. In our quantum description the corresponding solution delivers an attractive potential operating in the $\mathbb{R}_{6}$ space controlling the motion of the electron pair as a whole. That potential is given by

$$
W(R)=-\frac{g}{R^{3 / 2}}
$$

with the coupling constant

$$
g=\frac{\sqrt[4]{2}}{8}(\sqrt{100 Z-9}-\sqrt{4 Z-1})
$$

see (35). That result deserves several comments. First, a fractional power of $R$ in (36) is entirely unexpected. At a first glance one would expect the Coulomb law $-C_{0} / R$. But the diffraction pattern of the pair wave from the ridge leads to an $\mathbb{R}_{6}$ rotational symmetry breakdown and yields the surprising potential (42). The corresponding effective potential

$$
U(R)=\frac{15}{8 R^{2}}-\frac{g}{R^{3 / 2}}
$$

predicts a series of resonances below the double escape threshold. Such levels have been observed a long time ago by Cvejanovic and Read [3] in the system $\mathrm{He}^{-}$. The scattering of slow electrons from highly excited helium

$\mathrm{e}^{-}+\mathrm{He}(1 s, n s) \rightarrow \mathrm{He}^{-* *}$ showed a series of isolated lines below the ionization threshold of helium. A pilot analysis of their observed level spacing shows a decrease slower than in a Rydberg series. We believe that ref [3] shows only the upper part of the spectrum. The lower part of the eigenvalue spectrum of the potential (43) is covered over the regular spectral part described by the standard BO theory. Our analysis indicates a potential tail decreasing faster than a Coulomb potential. We conclude presently that the potential (42) is not inconsistent with the measurement [3].

\section{Conclusions}

Shell structure and circular orbits constitute since the early days of quantum mechanics the theoretical frame for multi-electron atoms although these terms stem from the exactly solvable hydrogen atom. Nevertheless models based on that theoretical frame have been surprisingly successful provided correlation is only a small effect. Near thresholds of multiple ionization, however, exist no longer circular orbits. They have been replaced by Wannier modes of motion.

Wannier [2] showed within classical mechanics that two slow highly excited electrons in nuclear field must be treated as one object in the larger pair-configuration space $\mathbb{R}_{6}$ rather than two electrons and each in a single-electron configuration 
space $\mathbb{R}_{3}$. Wannier's very unusual threshold cross section (1) let us suspect that a quantum version of that new description will discover more surprises.

The present paper presents the necessary quantum version, and opens an entirely new field of atomic physics full with surprises. We have shown that the new situation shortly referred to as Wannier phenomenon can be described with help of an amended BO approximation which replaces the set of BO potentials by an evolution operator. That operator delivers in fact an eigen-evolution which we refer to as Wannier channel. That channel delivers the threshold cross section (1), as well as a set of new isolated resonances slightly below threshold. They manifest themselves as eigenvalues of the potential $W(R)=-\frac{g}{R^{3 / 2}}$, see (42). Moreover, we show the Wannier evolution to be identical to a Fresnel distribution converging to a Dirac delta distribution centered at an equilibrium configuration of the electron pair. That corresponds to Wannier's converging trajectory which describes an electron-electron attraction. That attraction emerges from the diffraction of a dominantly correlated two-electron wave from a potential ridge. In summary we find out that the present work has confirmed our earlier suspiciousness on that topic [11].

Finally, we remark that this Wannier phenomenon worked out here can be extended to three/four excited electrons [4].

\section{Conflicts of Interest}

The author declares no conflicts of interest regarding the publication of this paper.

\section{References}

[1] Klar, H. (2020) The Born-Oppenheimer Approximation Revisited. Journal of Applied Mathematics and Physics, 8, 1507-1514. https://doi.org/10.4236/jamp.2020.88116

[2] Wannier, G.H. (1953) Ionization of an H Atom by an Electron at Threshold. Physical Review Journals Archive, 90, 857-875.

[3] Cvejanovic, S. and Read, F.S. (1974) Studies of the Electron Impact Ionization of Helium. Journal of Physics B: Atomic and Molecular Physics, 7, 1841. https://doi.org/10.1088/0022-3700/7/14/008

[4] Klar, H. (2018) Wave Propagation on a Potential Ridge. Physical Review Letters, 120, Article ID: 053401.https://doi.org/10.1103/PhysRevLett.120.053401

[5] Macek, J. H. (1968) Properties of Autoionizing States of Helium. Journal of Physics B: Atomic and Molecular Physics, 1, 831. https://doi.org/10.1088/0022-3700/1/5/309

[6] Klar, M. and Klar, H. (1980) An Accurate Treatment of Two-Electron Atoms in Hyperspherical Coordinates. Journal of Physics B: Atomic and Molecular Physics, 13, 1957-1072. https://doi.org/10.1088/0022-3700/13/6/014

[7] Sommerfeld, A. (1944) Atombau und Spektrallinien. Springer, Berlin.

[8] Born, M. and Oppenheimer, R. (1927) Zur Quantentheorie der Molekeln. Annalen der Physik, 389, 457-484. https://doi.org/10.1002/andp.19273892002

[9] See Any Textbook on Functional Analysis. 
[10] Fano, U. (1961) Effects of Configuration Interaction on Intensities and Phase Shifts. Physical Review Journals Archive, 124, 1866.

https://doi.org/10.1103/PhysRev.124.1866

[11] Klar, H. (2020) Dominant Correlation Effects in Two-Electron Atoms. Journal of Applied Mathematics and Physics, 8, 1424-1433.

https://doi.org/10.4236/jamp.2020.87108 